\title{
Primary Neuroendocrine Tumor of the Left Hepatic Duct: A Case Report with Review of the Literature
}

\author{
Ajay H. Bhandarwar, Taher A. Shaikh, Ashok D. Borisa, Jaydeep H. Palep, \\ Arun S. Patil, and Aditya A. Manke
} Division of GI and HPP Surgery, Department of Surgery, Grant Medical College \& Sir JJ Group of Hospitals, Byculla,
Mumbai 400008, India

Correspondence should be addressed to Ajay H. Bhandarwar, abhandarwar@yahoo.com

Received 29 April 2012; Accepted 29 August 2012

Academic Editors: T. Çolak and M. Ganau

Copyright ( $) 2012$ Ajay H. Bhandarwar et al. This is an open access article distributed under the Creative Commons Attribution License, which permits unrestricted use, distribution, and reproduction in any medium, provided the original work is properly cited.

Primary Biliary Tract Neuroendocrine tumors (NET) are extremely rare tumors with only 77 cases been reported in the literature till now. We describe a case of a left hepatic duct NET and review the literature for this rare malignancy. To the best of our knowledge the present case is the first reported case of a left hepatic duct NET in the literature. In spite of availability of advanced diagnostic tools like Computerized Tomography (CT) Scan and Endoscopic Retrograde Cholangio Pancreaticography (ERCP) a definitive diagnosis of these tumors is possible only after an accurate histopathologic diagnosis of operative specimens with immunohistochemistry and electron microscopy. Though surgical excision remains the gold standard treatment for such tumors, patients with unresectable tumors have good survival with newer biologic agents like Octreotride.

\section{Introduction}

NET is derived from the embryonal neural crest cells called Argentaffin or Kulchitsky cells and have a potential for secreting serotonin. This tumor can arise anywhere in the distribution of the Argentaffin cell system. In addition to the most common sites of occurrence, namely, ileum and appendix these tumors have reported to occur in bladder, prostate, rectum, stomach, bronchi, pancreas, and biliary tree.

Primary Biliary Tract NETs are rare and account for $0.2 \%-2 \%[1,2]$ of all gastrointestinal neuroendocrine tumors.

The Literature documents about 77 cases of neuroendocrine tumor arising from the biliary tree which includes common bile duct, common hepatic duct, cystic duct, and hilar confluence.

We report a case of Primary Biliary NET arising from the left hepatic duct. An extensive search of the literature has yielded no reference regarding a NET arising from the left Hepatic ducts. The present case is the first reported case of a NET arising in the left hepatic duct.

\section{Case Report}

A 69-years-old female presented with colicky pain in the right hypochondrium since 3 years. She had past history of open cholecystectomy done for gall stones 15 years back. On physical examination the patient was anicteric with soft abdomen. An Ultrasonography (USG) of the abdomen showed a hypoechoic lesion of size $3.5 \mathrm{~cm} \times 4 \mathrm{~cm}$ in segment 4 of the liver. Computerized Tomography (CT) of the abdomen showed a $4.1 \times 3.7 \mathrm{~cm}$ heterogeneously enhancing mass lesion in segment IV of liver abutting the left branch of portal vein (Figure 1). A Magnetic Resonance Imaging (MRI) of upper abdomen with Magnetic Resonance CholagioPancreaticography (MRCP) showed a filling defect in the left hepatic duct with lesion in the adjacent part of liver in segment IV, suggestive of a left hepatic duct tumor with infiltration in the liver (Figure 2). Tumor marker serum alfa fetoprotein was mildly raised. Patient was worked up for left hepatectomy. Intraopertaively a lesion arising from the left hepatic duct involving the left branch of portal vein and extending upto the portal confluence was found (Figure 3) rendering the tumor unresectable. A biopsy was 


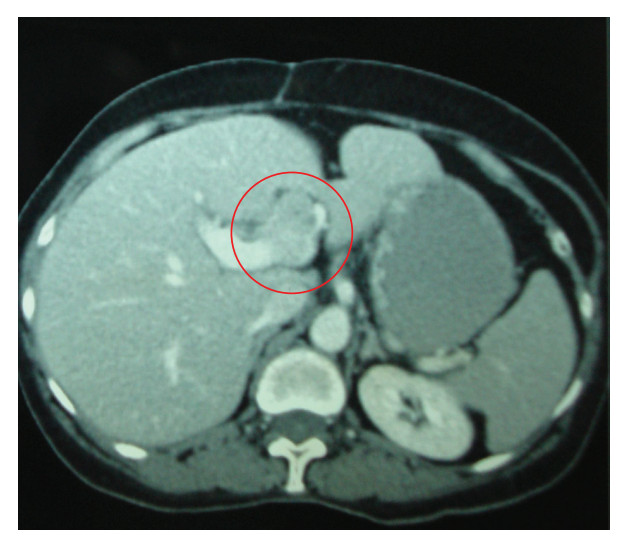

Figure 1: CT scan showing mass lesion in segment 4 abutting the left branch of portal vein.

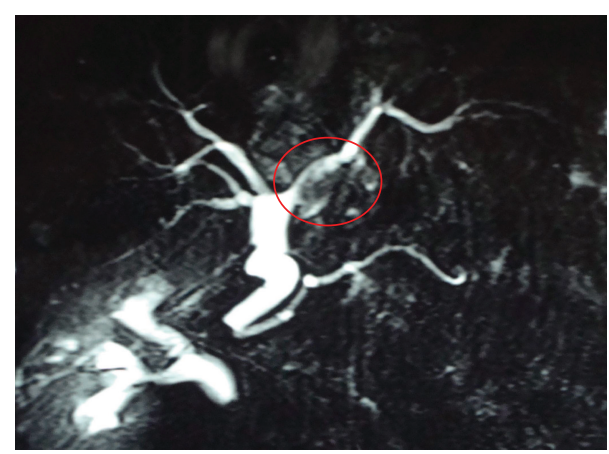

FIGURE 2: MRCP showing a filling defect in the left hepatic duct.

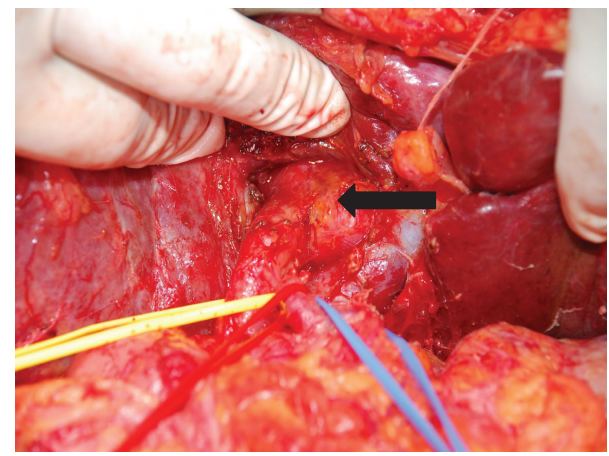

FIGURE 3: Intraoperative picture showing tumor arising from the left hepatic duct. CBD hooked in yellow, hepatic artery in red, and portal vein in blue.

taken from the mass and procedure was abandoned in view of inoperability. Histopathology showed typical rosette appearance of a neuroendocrine tumor (Figure 4) and immunohistochemistry positive for CD56, Chromogranin and Synaptophysin (Figure 5). Ultrastructural study of the cell with electron microscopy (Figure 6) showed the presence of multiple neurosecretory granules with muscle tissue.

A whole body Positron Emission Tomography (PET) scan and an Octreotride labeled radionulceotide scan showed
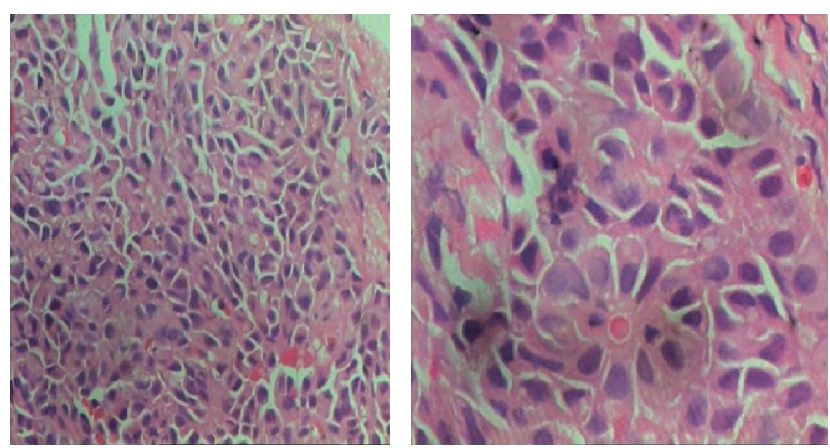

FIGURE 4: Histopathology showing a typical rosette appearance of neuroendocrine tumor.

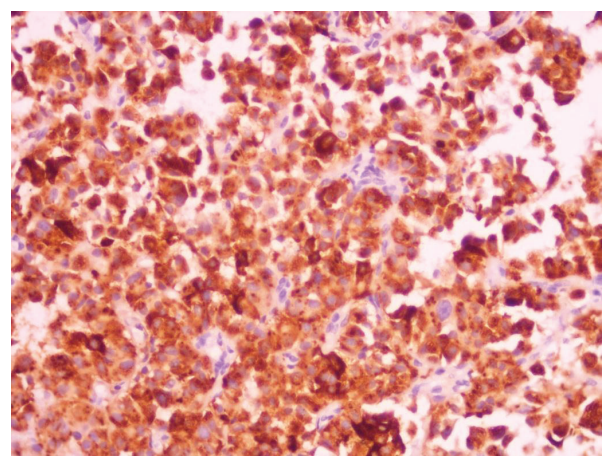

(a)

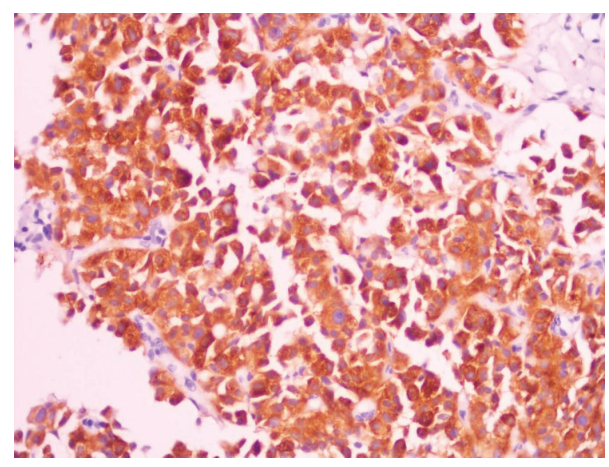

(b)

FIGURE 5: Immunohistochemistry staining positive for Chromogranin (a) and Synaptophysin (b).

somatostatin receptor expressing lesion in the hepatobiliary system (Figure 7).

The patient was started on long-acting Octreotride therapy single dose every month. The patient has received 12 of such doses and is doing well after a 1-year followup without any complications. A follow up MRI (Figure 8) upper abdomen with MRCP at 1 year showed the absence of filling defect in the Left hepatic duct that was seen previously which showed tumor regression. 

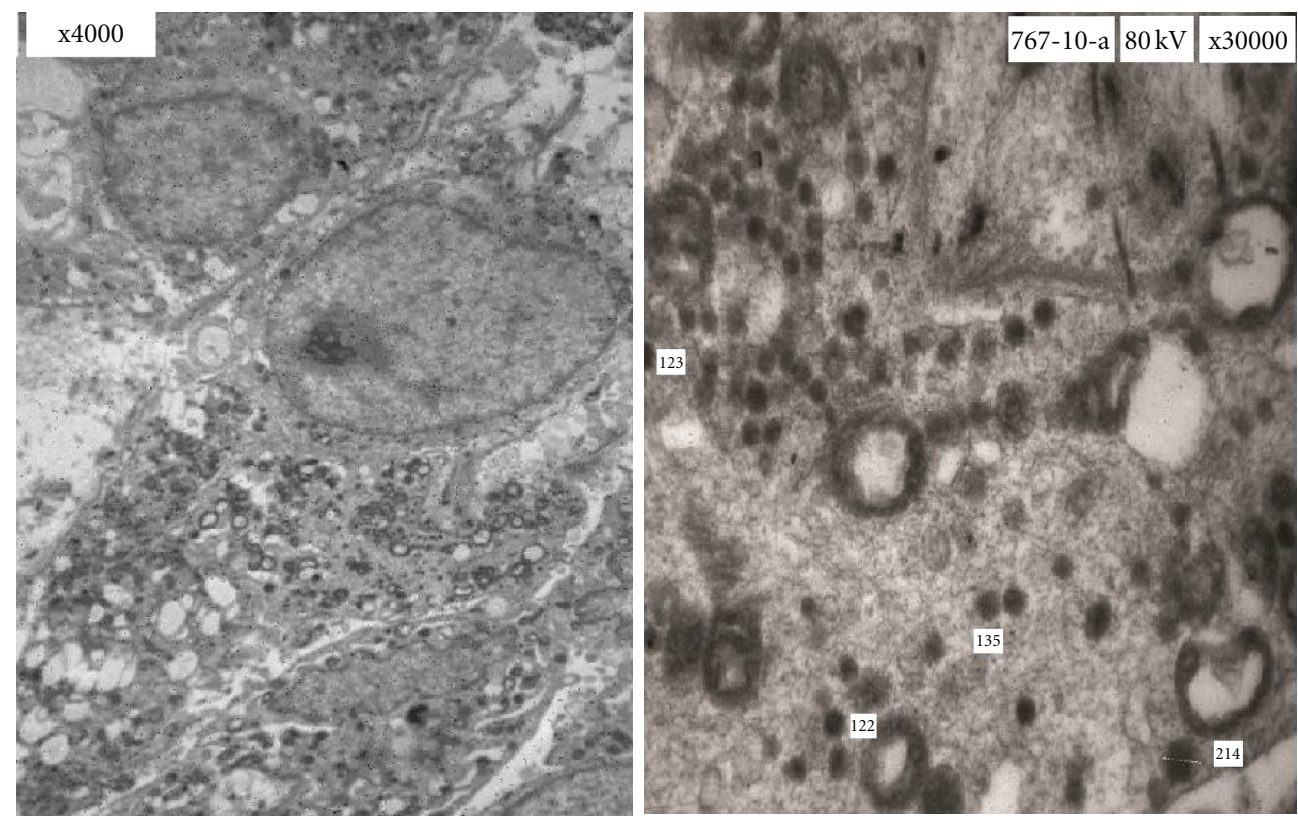

FIGURE 6: Electron microscopy picture showing multiple granules of varying sizes.

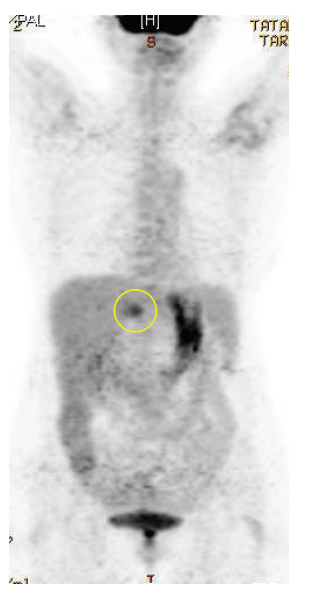

(a)

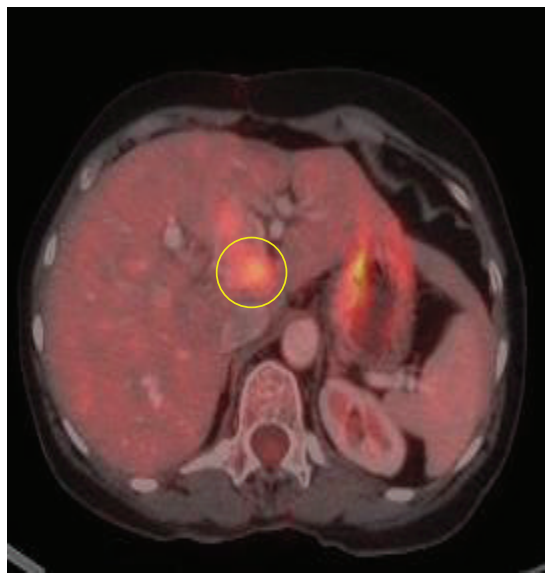

(b)

Figure 7: PET Scan (a) and Octreotride labelled (b) scan showing tumor limited to the hepatobiliary system.

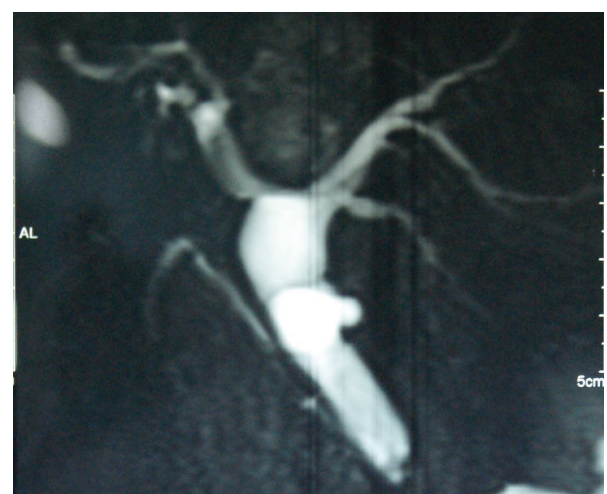

Figure 8: Follow up MRCP at 1 year showing absence of any filling defect in the left hepatic duct.

\section{Discussion}

Primary Biliary-tract NETs are very rare. They account for $0.2-2 \%$ of all gastrointestinal NET $[1,2]$ reason being the paucity of enterochromaffin cells from which NETs arise in this area. Chronic inflammation of the bile duct epithelium is responsible for metaplasia of these enterochromaffin cells and formation of NET.

Davies [3] in 1959 reported NET of the distal bile duct and pancreatic duct which represented more of a periampullary NET rather than a biliary tract. Pilz [4] in 1961 was credited to report the first case of a Biliary Tract NET. After an extensive search of the Medline only 77 cases of Biliary Tract NET have been reported so far in the literature since 1961 (Table 1).

Till now no NET in the literature has been reported in the isolated left hepatic duct possibly making our case the first reported case of an isolated left hepatic duct NET. 
TABLE 1: Showing study of reported cases of Biliary tract NET.

\begin{tabular}{|c|c|c|c|c|c|}
\hline No. & Case reference & Age & Sex & Complaint & Location \\
\hline (1) & Pilz, [4] 1961 & 55 & $\mathrm{~F}$ & Weakness & CBD \\
\hline (2) & $\begin{array}{c}\text { Little et al., [5] } \\
1968\end{array}$ & 41 & $\mathrm{~F}$ & $\begin{array}{l}\text { RUQ pain, } \\
\text { jaundice, }\end{array}$ & Hilar \\
\hline (3) & Bergdahl, [6] 1976 & 79 & $\mathrm{~F}$ & $\begin{array}{l}\text { Autopsy } \\
\text { finding }\end{array}$ & $\begin{array}{l}\text { Distal } \\
\text { CBD }\end{array}$ \\
\hline (4) & $\begin{array}{c}\text { Judge et al., [7] } \\
1976\end{array}$ & 19 & M & $\begin{array}{l}\text { Jaundice, } \\
\text { pain }\end{array}$ & Hilar \\
\hline (5) & $\begin{array}{c}\text { Gerlock and } \\
\text { Muhletaler [8] } \\
1979\end{array}$ & 32 & $\mathrm{M}$ & Jaundice & $\mathrm{CBD}$ \\
\hline (6) & $\begin{array}{c}\text { Vitaux et al., [9] } \\
1981\end{array}$ & 24 & $\mathrm{M}$ & Jaundice & $\begin{array}{l}\text { Distal } \\
\text { CBD }\end{array}$ \\
\hline (7) & $\begin{array}{c}\text { Nakamuara et al. } \\
\text { [10] } 1981\end{array}$ & 58 & $\mathrm{~F}$ & Jaundice & CBD \\
\hline (8) & $\begin{array}{c}\text { Abe et al., [11] } \\
1983\end{array}$ & 64 & M & RUQ pain & $\mathrm{CBD}$ \\
\hline (9) & $\begin{array}{l}\text { Goodman et al., } \\
\text { [12] } 1984\end{array}$ & 28 & $\mathrm{~F}$ & RUQ Pain & Cystic duct \\
\hline (10) & $\begin{array}{l}\text { Jutte et al., [13] } \\
1986\end{array}$ & 62 & M & Back Pain & $\mathrm{CHD}$ \\
\hline (11) & $\begin{array}{c}\text { Nicolescu and } \\
\text { Popescu, [14] } 1986\end{array}$ & 50 & $\mathrm{~F}$ & RUQ pain & CBD \\
\hline (12) & $\begin{array}{l}\text { Alexander et al., } \\
\text { [15] } 1986\end{array}$ & 64 & $\mathrm{~F}$ & Hematemesis & CBD \\
\hline (13) & $\begin{array}{c}\text { Gastinger et al. } \\
\text { [16] } 1987\end{array}$ & 65 & $\mathrm{~F}$ & $\begin{array}{l}\text { Jaundice, } \\
\text { Pain }\end{array}$ & Hilar \\
\hline (14) & $\begin{array}{c}\text { Reinhardt et al. } \\
\text { [17] } 1988\end{array}$ & 71 & $\mathrm{~F}$ & $\begin{array}{l}\text { Jaundice, } \\
\text { fever }\end{array}$ & $\mathrm{CBD}$ \\
\hline (15) & $\begin{array}{c}\text { Chittal and Ra, } \\
\text { [18] } 1989\end{array}$ & 46 & $\mathrm{~F}$ & RUQ pain & Cystic duct \\
\hline (16) & $\begin{array}{c}\text { Fujita et al., [19] } \\
1989\end{array}$ & 55 & $\mathrm{~F}$ & RUQ pain & $\mathrm{CBD}$ \\
\hline (17) & $\begin{array}{l}\text { Bickerstaff and } \\
\text { Ross [20] } 1989\end{array}$ & 57 & $\mathrm{~F}$ & Jaundice & CBD \\
\hline (18) & $\begin{array}{c}\text { Brown et al., [21] } \\
1990\end{array}$ & 35 & $\mathrm{~F}$ & Jaundice & Hilar \\
\hline (19) & $\begin{array}{c}\text { Bumin et al., [22] } \\
1990\end{array}$ & 38 & $\mathrm{~F}$ & Jaundice & CBD \\
\hline (20) & $\begin{array}{l}\text { Fellows et al. [23] } \\
1990\end{array}$ & 30 & $\mathrm{M}$ & Jaundice & CBD \\
\hline$(21)$ & Besznyák et al. [24] & 13 & $\mathrm{~F}$ & Jaundice & Hilar \\
\hline$(22)$ & $\begin{array}{l}\text { Angeles-Angeles et } \\
\text { al., [25] } 1991\end{array}$ & 39 & $\mathrm{~F}$ & Jaundice & $\mathrm{CBD}$ \\
\hline (23) & $\begin{array}{c}\text { Barron-Rodriguez } \\
\text { et al., [26] } 1991\end{array}$ & 36 & M & $\begin{array}{l}\text { Jaundice, } \\
\text { RUQ pain }\end{array}$ & CBD \\
\hline (24) & $\begin{array}{l}\text { Newman et al., } \\
1992 \text { [27] }\end{array}$ & 15 & $\mathrm{~F}$ & $\mathrm{~N} / \mathrm{A}$ & CBD \\
\hline$(25)$ & $\begin{array}{c}\text { Dixon et al., } 1992 \\
\text { [28] }\end{array}$ & 60 & $\mathrm{~F}$ & RUQ pain & CBD \\
\hline (26) & $\begin{array}{c}\text { Rugge et al., [29] } \\
1992\end{array}$ & 64 & $\mathrm{~F}$ & $\begin{array}{l}\text { Jaundice, } \\
\text { RUQ pain }\end{array}$ & $\begin{array}{l}\text { Cysticduct, } \\
\text { CBD }\end{array}$ \\
\hline (27) & $\begin{array}{l}\text { Gembala et al., } \\
\text { [30] } 1993\end{array}$ & 28 & M & Jaundice & Hilar \\
\hline (28) & $\begin{array}{c}\text { Mandujano-Vera } \\
\text { et al., [31] } 1995\end{array}$ & 53 & $\mathrm{~F}$ & Jaundice & $\mathrm{CBD}$ \\
\hline (29) & $\begin{array}{c}\text { Sankary et al., [32] } \\
1995\end{array}$ & 47 & $\mathrm{~F}$ & Jaundice & Hilar \\
\hline
\end{tabular}

TABle 1: Continued.

\begin{tabular}{|c|c|c|c|c|c|}
\hline No. & Case reference & Age & Sex & Complaint & Location \\
\hline$(30)$ & $\begin{array}{l}\text { Hao et al., [33] } \\
1996\end{array}$ & 47 & M & $\begin{array}{l}\text { Incidental } \\
\text { finding }\end{array}$ & CBD \\
\hline$(31)$ & $\begin{array}{l}\text { Kopelman et al., } \\
\text { [34] } 1996\end{array}$ & 44 & M & Jaundice & $\mathrm{CBD}$ \\
\hline$(32)$ & $\begin{array}{l}\text { Belli et al., [35] } \\
1996 \text { CBD }\end{array}$ & 78 & M & Jaundice & CBD \\
\hline (33) & $\begin{array}{c}\text { Bembenek et al., } \\
\text { [36] } 1998\end{array}$ & 12 & $\mathrm{~F}$ & Jaundice & Hilar \\
\hline$(34)$ & $\begin{array}{c}\text { Nahas et al., [37] } \\
1998\end{array}$ & 61 & $\mathrm{~F}$ & Jaundice & Hilar \\
\hline (35) & $\begin{array}{c}\text { Ross et al., [38] } \\
1999\end{array}$ & 65 & $\mathrm{~F}$ & Jaundice & CBD \\
\hline$(36)$ & $\begin{array}{c}\text { Chamberlain and } \\
\text { Blumgart [39] } \\
1999\end{array}$ & 37 & $\mathrm{~F}$ & Itching & Hilar \\
\hline$(37)$ & $\begin{array}{c}\text { Chamberlain and } \\
\text { Blumgart [39] } \\
1999\end{array}$ & 67 & $\mathrm{~F}$ & Itching & Hilar \\
\hline$(38)$ & $\begin{array}{l}\text { Hermina et al., } \\
\text { [40] } 1999\end{array}$ & 69 & M & RUQ pain & Cystic duct \\
\hline (39) & $\begin{array}{l}\text { Perakath et al. [42] } \\
1999\end{array}$ & 36 & $\mathrm{~F}$ & $\begin{array}{l}\text { Jaundice, } \\
\text { Pain }\end{array}$ & $\mathrm{CHD}$ \\
\hline$(40)$ & $\begin{array}{l}\text { Chan et al [41] } \\
2000\end{array}$ & 14 & M & Jaundice & Hilar \\
\hline$(41)$ & $\begin{array}{l}\text { Maitra et al., [42] } \\
\qquad 2000\end{array}$ & 53 & $\mathrm{~F}$ & Jaundice, & CBD \\
\hline$(42)$ & $\begin{array}{l}\text { Maitra et al., [42] } \\
2000\end{array}$ & 61 & $\mathrm{~F}$ & $\begin{array}{l}\text { Jaundice, } \\
\text { itching }\end{array}$ & Hilar \\
\hline$(43)$ & $\begin{array}{c}\text { Juturi et al., [43] } \\
2000\end{array}$ & 43 & M & $\begin{array}{l}\text { Jaundice, } \\
\text { itching }\end{array}$ & CBD \\
\hline$(44)$ & $\begin{array}{l}\text { Turrión et al., [44] } \\
\qquad 2002\end{array}$ & 51 & $\mathrm{~F}$ & $\begin{array}{l}\text { Jaundice, } \\
\text { itching }\end{array}$ & Hilar \\
\hline$(45)$ & $\begin{array}{l}\text { Pawlik et al., [45] } \\
2003\end{array}$ & 59 & M & Jaundice & Hilar \\
\hline$(46)$ & $\begin{array}{l}\text { Podnos et al., [46] } \\
2003\end{array}$ & 65 & $\mathrm{~F}$ & Cholecystitis & CBD \\
\hline (47) & $\begin{array}{l}\text { Podnos et al., [46] } \\
2003\end{array}$ & 27 & M & $\begin{array}{l}\text { Jaundice, } \\
\text { itching }\end{array}$ & $\mathrm{CBD}$ \\
\hline$(48)$ & $\begin{array}{l}\text { Volpe et al., [47] } \\
\qquad 2003\end{array}$ & 19 & M & $\begin{array}{l}\text { Jaundice, } \\
\text { pain }\end{array}$ & $\mathrm{CBD}$ \\
\hline$(49)$ & Menezes et al., [48] & 30 & M & Jaundice & $\mathrm{CHD}$ \\
\hline (50) & $\begin{array}{l}\text { Ligato et al., [49] } \\
\qquad 2005\end{array}$ & 33 & $\mathrm{~F}$ & $\begin{array}{c}\text { Irritable } \\
\text { bowel }\end{array}$ & Hilar \\
\hline (51) & $\begin{array}{l}\text { Hubert et al., [50] } \\
\qquad 2005\end{array}$ & NA & M & Jaundice & CBD \\
\hline (52) & $\begin{array}{l}\text { Hubert et al., [50] } \\
\qquad 2005\end{array}$ & NA & M & Jaundice & CBD \\
\hline (53) & $\begin{array}{l}\text { Hubert et al., [50] } \\
2005\end{array}$ & NA & $\mathrm{F}$ & Jaundice & CBD \\
\hline (54) & $\begin{array}{l}\text { Nesi et al., [51] } \\
2006\end{array}$ & 30 & M & $\begin{array}{l}\text { Jaundice, } \\
\text { iarrhoea }\end{array}$ & CBD \\
\hline (55) & $\begin{array}{l}\text { Kim et al., [52] } \\
2006\end{array}$ & 67 & $\mathrm{~F}$ & Jaundice & CBD \\
\hline (56) & $\begin{array}{l}\text { Caglikulekci et al., } \\
\text { [53] } 2006\end{array}$ & 40 & $\mathrm{~F}$ & Jaundice & Hilar \\
\hline (57) & $\begin{array}{l}\text { Honda et al., [54] } \\
2006\end{array}$ & 76 & $\mathrm{M}$ & $\begin{array}{l}\text { Jaundice, } \\
\text { pain }\end{array}$ & CBD \\
\hline
\end{tabular}


TABle 1: Continued.

\begin{tabular}{|c|c|c|c|c|c|}
\hline No. & Case reference & Age & Sex & Complaint & Location \\
\hline (58) & $\begin{array}{l}\text { Todorki et al., [55] } \\
2007\end{array}$ & 73 & M & $\begin{array}{l}\text { Jaundice, } \\
\text { Fever }\end{array}$ & CBD \\
\hline$(59)$ & $\begin{array}{l}\text { Sethi et al., [56] } \\
\qquad 2007\end{array}$ & 51 & M & ERCP finding & $\begin{array}{c}\text { CHD with } \\
\text { Cystic }\end{array}$ \\
\hline$(60)$ & $\begin{array}{l}\text { Stavridi et al., [57] } \\
2007\end{array}$ & NA & NA & NA & Cystic duct \\
\hline (61) & $\begin{array}{l}\text { Jiménez et al., [58] } \\
2007\end{array}$ & 60 & M & Jaundice & Hilar \\
\hline$(62)$ & $\begin{array}{l}\text { Ferrone et al., [59] } \\
\qquad 2007\end{array}$ & NA & NA & NA & NA \\
\hline$(63)$ & $\begin{array}{l}\text { Nafidi et al., [60] } \\
2008\end{array}$ & 31 & $\mathrm{~F}$ & RUQ pain & CBD \\
\hline$(64)$ & $\begin{array}{l}\text { Gusani et al., [61] } \\
2008\end{array}$ & NA & NA & NA & $\mathrm{CBD}$ \\
\hline$(65)$ & $\begin{array}{l}\text { Schmitt et al., [62] } \\
2008\end{array}$ & NA & NA & NA & Hilar \\
\hline$(66)$ & $\begin{array}{l}\text { Costantini et al., } \\
\text { [63] } 2008\end{array}$ & NA & NA & NA & $\mathrm{CHD}$ \\
\hline$(67)$ & $\begin{array}{l}\text { Felekouras et al., } \\
\qquad[64] 2009\end{array}$ & 60 & $\mathrm{~F}$ & Jaundice & Cystic duct \\
\hline$(68)$ & $\begin{array}{l}\text { Price et al., [65] } \\
2009\end{array}$ & NA & NA & Jaundice & CBD \\
\hline$(69)$ & $\begin{array}{l}\text { Price et al., [65] } \\
2009\end{array}$ & NA & NA & Jaundice & CBD \\
\hline$(70)$ & $\begin{array}{l}\text { Price et al., [65] } \\
2009\end{array}$ & NA & NA & Jaundice & Hilar \\
\hline$(71)$ & $\begin{array}{c}\text { Tonnhofer et al., } \\
{[66]}\end{array}$ & 6 & $\mathrm{~F}$ & Jaundice & $\mathrm{CBD}$ \\
\hline$(72)$ & $\begin{array}{l}\text { Zhan et al. [67] } \\
2010\end{array}$ & 10 & M & NA & $\mathrm{CBD}$ \\
\hline (73) & $\begin{array}{l}\text { Squillaci et al., [68] } \\
\qquad 2010\end{array}$ & 52 & M & Jaundice & CBD \\
\hline$(74)$ & $\begin{array}{l}\text { Squillaci et al., [68] } \\
2010\end{array}$ & 70 & M & Jaundice & $\mathrm{CBD}$ \\
\hline$(75)$ & $\begin{array}{c}\text { Tsalis et al., [69] } \\
2010\end{array}$ & 77 & M & Incidental & Hilar \\
\hline$(76)$ & Lee et al., [70] 2011 & 59 & M & Jaundice & CBD \\
\hline (77) & $\begin{array}{l}\text { Athanasopoulos et } \\
\text { al., [71] } 2011\end{array}$ & 43 & M & Jaundice & $\mathrm{CBD}$ \\
\hline (78) & Present case & 69 & $\mathrm{~F}$ & Pain & $\begin{array}{c}\text { Left } \\
\text { hepatic } \\
\text { duct }\end{array}$ \\
\hline
\end{tabular}

CHD: common hepatic duct, CBD: common bile duct, Hilar at the common bile duct bifurcation.

The most common site of malignancy in the biliary tract was common bile duct $(57.14 \%)$ followed by the hilar confluence $(27.28 \%)$, the cystic duct $(9.1 \%)$, common hepatic duct (5.12\%) and finally the left hepatic duct $(1.23 \%)$.

The mean age of presentation was 47 years (range 6 years to 79 years).

The male to female ratio is $1: 1.23$ showing that the biliary NET has a preponderance for female.
By far the most common symptom in patients of Biliary tract NET is Jaundice (63.4\%) followed by Pain (14.1\%), jaundice with pain $(12.7 \%)$ and remaining nonspecific symptoms like weight loss.

The incidence of a Carcinoid syndrome in patients of Biliary Tract NET is very rare. Only 4 cases which include a single case published by Nesi et al. [51] in 2006 with symptoms of diarrhea due to secretion of serotonin and 3 cases by Price et al. [65] in 2009 with features of Zollinger Ellison syndrome due to secretion of gastrin from tumor in CBD.

\section{Conclusion}

Biliary Tract NET are rare tumors that typically present with jaundice and pain. As compared to its counterpart Cholangiocarcinoma Biliary NET occurs in a younger age group with a female preponderance [39]. Biliary NET usually are nonsecreting tumor. Preoperative diagnosis of these tumors require a high index of suspicion and accurate histopathological diagnosis which must include a immunohistochemistry study and electron microscopy. Biliary tract NET are slowgrowing indolent tumor which have a limited propensity for local and metastatic spread. Surgical resection aimed at complete tumor excision with bilio-enteric continuity offers the best cure and high survival rates. Patients who have undergone resection have a long term survival. Even in inoperable patients chemotherapy with newer biologic agents like Octreotride have a favorable outcome on the patient's survival.

\section{Conflict of Interests}

The authors declare that they have no conflict of interest.

\section{Acknowledgments}

We would like to thank Padmashree Dr. T. P. Lahane Dean of Grant Medical College Mumbai for the support and encouragement provided to publish this paper. We would also like to thank Dr. Sanjay Navani and Dr. M. B. Tayade Head of department General Surgery Grant Medical College Mumbai.

\section{References}

[1] I. M. Modlin and A. Sandor, "An analysis of 8305 cases of Carcinoid tumors," Cancer, vol. 79, no. 4, pp. 813-829, 1997.

[2] J. M. Lauffer, T. Zhang, and I. M. Modlin, "Review article:current status of gastrointestinal carcinoids," Alimentary Pharmacology \& Therapeutics, vol. 13, no. 3, pp. 271-287, 1999.

[3] A. J. Davies, "Carcinoid tumors (Argentaffinomata)," Annals of the Royal College of Surgeons of England, vol. 25, pp. 277-297, 1959.

[4] E. Pilz, "On carcinoid of the bile duct," Zentralblatt Fur Chirurgie, vol. 86, pp. 1588-1590, 1961. 
[5] J. M. Little, A. A. Gibson, and A. W. Kay, "Primary common bile-duct carcinoid," British Journal of Surgery, vol. 55, no. 2, pp. 147-149, 1968.

[6] L. Bergdahl, "Carcinoid tumours of the biliary tract," Australian and New Zealand Journal of Surgery, vol. 46, no. 2, pp. 136-138, 1976.

[7] D. M. Judge, P. S. Dickman, and S. Trapukdi, "Nonfunctioning argyrophilic tumor (APUDoma) of the hepatic duct. Simplified methods of detecting biogenic amines in tissue," American Journal of Clinical Pathology, vol. 66, no. 1, pp. 40-45, 1976.

[8] A. J. Gerlock and C. A. Muhletaler, "Primary common bile duct carcinoid," Gastrointestinal Radiology, vol. 4, no. 3, pp. 263-264, 1979.

[9] J. Vitaux, R. J. Salmon, O. Langville, C. Buffet, E. Martin, and J. C. Chaput, "Carcinoid tumor of the common bile duct," American Journal of Gastroenterology, vol. 76, no. 4, pp. 360362, 1981.

[10] T. Nakamuara, T. Sawada, K. Karaki, and A. Kawao :, "A case of MEA associated with bie duct carcinoid," Gann No Rinnshou, vol. 27, pp. 1299-1301, 1981.

[11] Y. Abe, H. Itoh, M. Yoshida et al., "Carcinoid tumor of the common bile duct, report of a case," Stomach and Intestine, vol. 18 , no. 5, pp. 543-548, 1983.

[12] Z. D. Goodman, J. Albores Saavedra, and D. M. Lundblad, "Somatostatinoma of the cystic duct," Cancer, vol. 53, no. 3, pp. 498-502, 1984.

[13] D. L. Jutte, P. H. Bell, I. M. Penn, J. Powers, and J. Kolinjivadi, "Carcinoid tumor of the biliary system," Digestive Diseases and Sciences, vol. 32, pp. 763-769, 1986.

[14] P. G. Nicolescu and A. Popescu, "Carcinoid tumor of the cystic duct," Morphologie et Embryologie, vol. 32, no. 4, pp. 275-277, 1986.

[15] I. A. Alexander, K. K. Thomson, and G. A. Klune, "Primarycommon bile duct carcinoid: demonstration by computed tomography, ultrasonography and angiography," Australasian Radiology, vol. 30, pp. 34-37, 1986.

[16] I. Gastinger, U. Schütze, G. Beetz, and H. Lippert, "Obstructive jaundice caused by a carcinoid tumor of the hepatocholedochal duct," Zentralblatt fur Chirurgie, vol. 112, no. 18, pp. 1170-1175, 1987.

[17] D. Reinhardt, H. Thiele, J. C. Weber, and W. J. Hofmann, "Carcinoid of the ductus choledochus. A rare cause of obstructive jaundice," Chirurg, vol. 59, no. 10, pp. 683-687, 1988.

[18] S. M. Chittal and P. M. Ra, "Carcinoid of the cystic duct," Histopathology, vol. 15, no. 6, pp. 643-646, 1989.

[19] N. Fujita, F. Mochizuki, S. Lee et al., "Carcinoid tumor of the bile duct: case report," Gastrointestinal Radiology, vol. 14, no. 2, pp. 151-154, 1989.

[20] D. R. Bickerstaff and W. B. Ross, "Carcinoid of the biliary tree: a case report and review of the literature," Journal of the Royal College of Surgeons of Edinburgh, vol. 32, no. 1, pp. 48-51, 1987.

[21] W. M. Brown, J. M. Henderson, and J. C. Kennedy, "Carcinoid tumor of the bile duct. A case report and literature review," American Surgeon, vol. 56, no. 6, pp. 343-346, 1990.

[22] C. Bumin, N. Ormeci, M. Dolapci, and S. Gungor, "Carcinoid tumor of the biliary duct," International Surgery, vol. 75, no. 4, pp. 262-264, 1990.

[23] I. W. Fellows, I. H. Leach, P. G. Smith, P. J. Toghill, and J. Doran, "Carcinoid tumour of the common bile duct-a novel complication of von Hippel-Lindau syndrome," Gut, vol. 31, no. 6, pp. 728-729, 1990.
[24] I. Besznyák, G. Tasnádi, T. Hirsch, I. Köves, Z. Schaff, and K. Lapis, "Hepato-lobectomy for carcinoid tumor of the hepatic duct," Orvosi Hetilap, vol. 132, no. 11, pp. 591-594, 1991.

[25] A. Angeles-Angeles, L. Quintanilla-Martinez, and J. LarrivaSahd, "Primary carcinoid of the common bile duct. Immunohistochemical characterization of a case and review of the literature," American Journal of Clinical Pathology, vol. 96, no. 3, pp. 341-344, 1991.

[26] L. P. Barron-Rodriguez, J. C. Manivel, N. Mendez-Sanchez, and J. Jessurun, "Carcinoid tumor of the common bile duct: evidence for its origin in metaplastic endocrine cells," American Journal of Gastroenterology, vol. 86, no. 8, pp. 10731076, 1991.

[27] K. Newman, T. Vates, L. Duffy, and K. Anderson, "Pancreatoduodenectomy with preservation of the stomach and pylorus: a safe and effective alternative in children," Journal of Pediatric Surgery, vol. 27, no. 10, pp. 1334-1335, 1992.

[28] J. M. Dixon, R. W. Chapman, and A. R. Berry, "Carcinoid tumour of the ampulla of Vater presenting as acute pancreatitis," Gut, vol. 28, no. 10, pp. 1296-1297, 1987.

[29] M. Rugge, F. Sonego, C. Militello, M. Guido, and V. Ninfo, "Primary carcinoid tumor of the cystic and common bile ducts," American Journal of Surgical Pathology, vol. 16, no. 8, pp. 802-807, 1992.

[30] R. B. Gembala, J. E. Arsuaga, A. C. Friedman et al., "Carcinoid of the intrahepatic ducts," Abdominal Imaging, vol. 18, no. 3, pp. 242-244, 1993.

[31] G. Mandujano-Vera, A. Angeles-Angeles, J. De la CruzHernandez, M. Sansores- Perez, and J. Larriva-Sahd, "Gastrinoma of the common bile duct: immunohistochemical and ultrastructural study of a case," Journal of Clinical Gastroenterology, vol. 20, no. 4, pp. 321-324, 1995.

[32] H. N. Sankary, P. Foster, E. Frye, and J. W. Williams, "Carcinoid tumors of the extrahepatic bile duct: an unusual cause of bile duct obstruction," Liver Transplantation and Surgery, vol. 1, no. 2, pp. 122-123, 1995.

[33] L. Hao, A. L. Friedman, V. J. Navarro, B. West, and M. E. Robert, "Carcinoid tumor of the common bile duct producing gastrin and serotonin," Journal of Clinical Gastroenterology, vol. 23, no. 1, pp. 63-65, 1996.

[34] D. Kopelman, M. Schein, H. Kerner, H. Bahuss, and M. Hashmonai, "Carcinoid tumor of the common bile duct," $H P B$ Surgery, vol. 10, no. 1, pp. 41-43, 1996.

[35] G. Belli, G. Rotondano, A. D’Agostino, and I. Marano, "Primary extrahepatic bile duct carcinoids," HPB Surgery, vol. 9, no. 2, pp. 101-105, 1996.

[36] A. Bembenek, E. Lotterer, A. Machens et al., "Neuroendocrine tumor of the common heaptic duct: a rare cause of extrahepatic jaundice in adolescence," Surgery, vol. 123, no. 6, pp. 712715, 1998.

[37] S. C. Nahas, J. L. Lourencao, E. Gazoni, C. Mott de B, M. R. Borba, J. O. Pinto Filho et al., "Carcinoid tumors of the common bile duct: report of a case," Revista Do Hospital Das Clinicas Da Faculdade De Medicina Da Universidade De Sao Paulo, vol. 53, pp. 26-28, 1998.

[38] A. C. Ross, J. B. Hurley, W. B. Hay, C. H. Rusnak, and D. M. Petrunia, "Carcinoids of the common bile duct: a case report and literature review," Canadian Journal of Surgery, vol. 42, no. 1, pp. 59-63, 1999.

[39] R. S. Chamberlain and L. H. Blumgart, "Carcinoid tumors of the extrahepatic bile duct. A rare cause of malignant biliary obstruction," Cancer, vol. 86, pp. 1959-1965, 1999. 
[40] M. Hermina, J. Starling, and T. F. Warner, "Carcinoid tumor of the cystic duct," Pathology Research and Practice, vol. 195, no. 10, pp. 707-709, 1999.

[41] C. Chan, H. Medina-Franco, W. Bell, A. Lazenby, and S. M. Vickers, "Carcinoid tumor of the hepatic duct presenting as a Klatskin tumor in an adolescent and review of world literature," Hepato-Gastroenterology, vol. 47, no. 32, pp. 519$521,2000$.

[42] A. Maitra, J. E. Krueger, M. Tascilar et al., "Carcinoid tumors of the extrahepatic bile ducts: a study of seven cases," American Journal of Surgical Pathology, vol. 24, no. 11, pp. 1501-1510, 2000.

[43] J. V. Juturi, I. Maghfoor, D. C. Doll, and M. L. Evans, "A case of biliary carcinoid presenting with pancreatitis and obstructive jaundice," American Journal of Gastroenterology, vol. 95, no. 10, pp. 2973-2974, 2000.

[44] V. S. Turrión, C. Salas, L. G. Alvira, M. Jimenez, J. L. Lucena, and J. Ardaiz, "Carcinoid tumour of the common bile duct: an exceptional indication for liver transplantation," Transplantation Proceedings, vol. 34, no. 1, pp. 264-265, 2002.

[45] T. M. Pawlik, S. Shah, and F. E. Eckhauser, "Carcinoid tumor of the biliary tract: treating a rare cause of bile duct obstruction," American Surgeon, vol. 69, no. 2, pp. 98-101, 2003.

[46] Y. D. Podnos, J. C. Jimenez, K. Zainabadi et al., "Carcinoid tumors of the common bile duct: report of two cases," Surgery Today, vol. 33, no. 7, pp. 553-555, 2003.

[47] C. M. Volpe, J. P. Pryor, M. Caty, and R. J. Doerr, "An adolescent with bile duct carcinoid tumor," Medical and Pediatric Oncology, vol. 40, no. 2, pp. 137-138, 2003.

[48] A. A. C. Menezes, A. J. Diver, D. McCance, and T. Diamond, "Carcinoid tumour of the extrahepatic bile duct-report of a case and literature review," Ulster Medical Journal, vol. 73, no. 1, pp. 59-62, 2004.

[49] S. Ligato, W. Furmaga, R. W. Cartun, D. Hull, and G. J. Tsongalis, "Primary carcinoid tumor of the common hepatic duct: a rare case with immunohistochemical and molecular findings," Oncology Reports, vol. 13, no. 3, pp. 543-546, 2005.

[50] C. Hubert, C. Sempoux, A. Berquin, P. Deprez, F. Jamar, and J. F. Gigot, "Bile duct carcinoid tumors: an uncommon disease but with a good prognosis?" Hepato-Gastroenterology, vol. 52, no. 64, pp. 1042-1047, 2005.

[51] G. Nesi, A. Lombardi, G. Batignani, F. Ficari, C. A. Rubio, and F. Tonelli, "Well-differentiated endocrine tumor of the distal common bile duct: a case study and literature review," Virchows Archiv, vol. 449, no. 1, pp. 104-111, 2006.

[52] D. H. Kim, M. H. Song, and D. H. Kim, "Malignant carcinoid tumor of the common bile duct: report of a case," Surgery Today, vol. 36, no. 5, pp. 485-489, 2006.

[53] M. Caglikulekci, M. Dirlik, O. Aydin et al., "Carcinoid tumour of the common bile duct: report of a case and a review of the literature," Acta Chirurgica Belgica, vol. 106, no. 1, pp. 112$115,2006$.

[54] H. Honda, S. Hayashi, Y. Sekiguchi, T. Tsukadaira, and K. Nakamura, "A case of the extrahepatic bile duct carcinoid tumor," Nihon Shokakibyo Gakkai Zasshi, vol. 103, no. 10, pp. 1169-1175, 2006.

[55] T. Todoroki, T. Sano, S. Yamada et al., "Clear cell carcinoid tumor of the distal common bile duct," World Journal of Surgical Oncology, vol. 5, article 6, 2007.

[56] H. Sethi, M. Madanur, P. Srinivasan, B. Portmann, N. Heaton, and M. Rela, "Non-functioning well-differentiated neuroendocrine tumor of the extrahepatic bile duct: an unusual suspect?" Hepatobiliary and Pancreatic Diseases International, vol. 6, no. 5, pp. 549-552, 2007.
[57] F. Stavridi, H. Chong, S. Chan et al., "Neuroendocrine tumour of the cystic duct: a case report and literature review," Journal of Gastrointestinal Cancer, vol. 38, no. 1, pp. 32-33, 2007.

[58] R. Jiménez, A. Beguiristain, I. Ruiz-Montesinos et al., "Intrahepatic biliary carcinoid," American Journal of Clinical Oncology, vol. 31, no. 5, pp. 521-522, 2008.

[59] C. R. Ferrone, L. H. Tang, M. D’Angelica et al., "Extrahepatic bile duct carcinoid tumors: malignant biliary obstruction with a good prognosis," Journal of the American College of Surgeons, vol. 205, no. 2, pp. 357-361, 2007.

[60] O. Nafidi, B. N. Nguyen, and A. Roy, "Carcinoid tumor of the common bile duct: a rare complication of von Hippel-Lindau syndrome," World Journal of Gastroenterology, vol. 14, no. 8, pp. 1299-1301, 2008.

[61] N. J. Gusani, J. W. Marsh, M. A. Nalesnik, M. E. Tublin, and T. C. Gamblin, "Carcinoid of the extra-hepatic bile duct: a case report with long-term follow-up and review of literature," American Surgeon, vol. 74, no. 1, pp. 87-90, 2008.

[62] T. M. Schmitt, H. Bonatti, K. D. Hagspiel, J. Iezzoni, P. Northup, and T. L. Pruett, "Carcinoid of the bile duct bifurcation," Journal of the American College of Surgeons, vol. 206, no. 2, p. 399, 2008.

[63] M. Costantini, R. Montalti, G. Rossi et al., "Adenocarcinoid tumor of the extrahepatic biliary tract," International Journal of Surgical Pathology, vol. 16, no. 4, pp. 455-457, 2008.

[64] E. Felekouras, A. Petrou, K. Bramis et al., "Malignant carcinoid tumor of the cystic duct: a rare cause of bile duct obstruction," Hepatobiliary and Pancreatic Diseases International, vol. 8, no. 6, pp. 640-646, 2009.

[65] T. N. Price, G. B. Thompson, J. T. Lewis, R. V. Lloyd, and W. F. Young, "Zollinger-Ellison syndrome due to primary gastrinoma of the extrahepatic biliary tree: three case reports and review of literature," Endocrine Practice, vol. 15, no. 7, pp. 737-749, 2009.

[66] U. Tonnhofer, C. Balassy, C. A. Reck, A. Koller, and E. Horcher, "Neuroendocrine tumor of the common hepatic duct, mimicking a choledochal cyst in a 6-year-old child," Journal of Pediatric Surgery, vol. 44, no. 6, pp. e23-e25, 2009.

[67] J. Zhan, G. Bao, X. Hu et al., "Carcinoid tumor of the common bile duct in children: a case report," Journal of Pediatric Surgery, vol. 45, no. 10, pp. 2061-2063, 2010.

[68] S. Squillaci, R. Marchione, M. Piccolomini et al., "Welldifferentiated neuroendocrine carcinoma (malignant carcinoid) of the extrahepatic biliary tract: report of two cases and literature review," APMIS, vol. 118, no. 8, pp. 543-556, 2010.

[69] K. Tsalis, G. Vrakas, T. Geroukis, A. Cheva, G. N. Roidos, and C. Lazarides, "Primary neuroendocrine tumor of the extrahepatic biliary tree mimicking Klatskin tumor," Journal of Gastrointestinal and Liver Diseases, vol. 19, no. 3, pp. 341$342,2010$.

[70] J. H. Lee, K. G. Lee, Y. H. Oh, S. S. Paik, H. K. Park, and K. S. Lee, "Carcinoid tumors of the extrahepatic biliary tract: report of four cases," Surgery Today, vol. 41, no. 3, pp. 430-435, 2011.

[71] P. G. Athanasopoulos, N. Arkadopoulos, V. Stafyla et al., "A rare combination of an endocrine tumour of the common bile duct and a follicular lymphoma of the ampulla of Vater: a case report and review of the literature," World Journal of Surgical Oncology, vol. 9, article 4, 2011. 


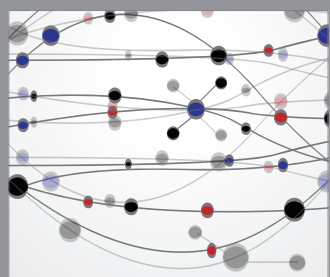

The Scientific World Journal
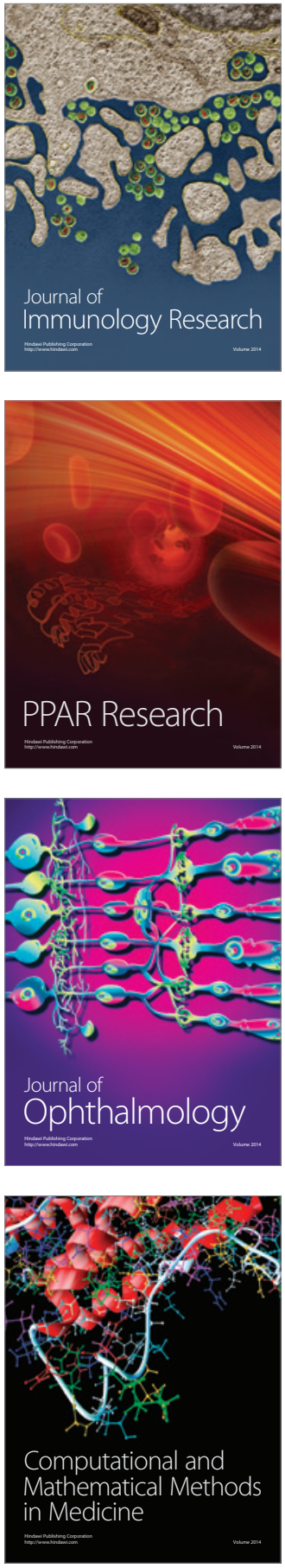

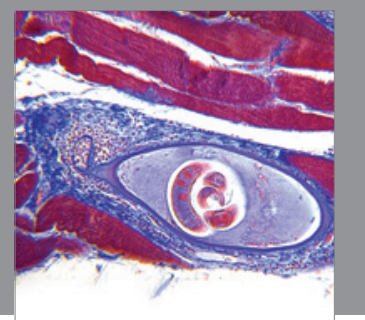

Gastroenterology

Research and Practice
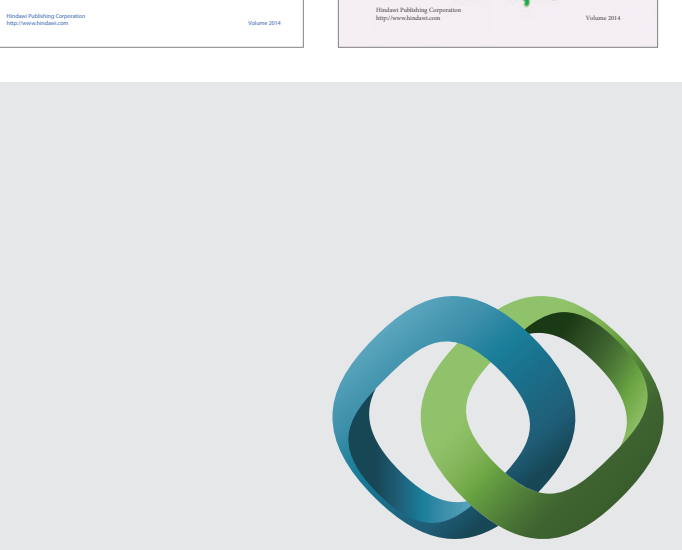

\section{Hindawi}

Submit your manuscripts at

http://www.hindawi.com
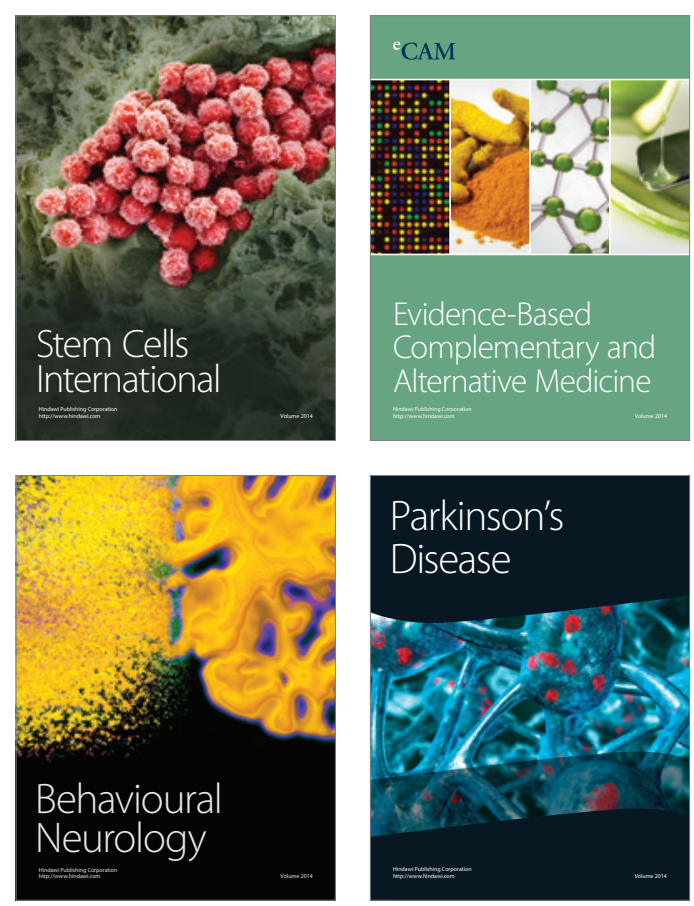

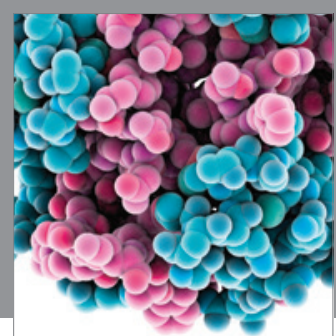

Journal of
Diabetes Research

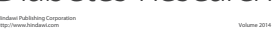

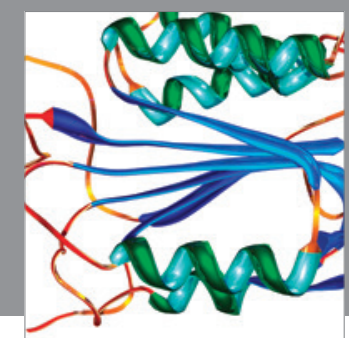

Disease Markers
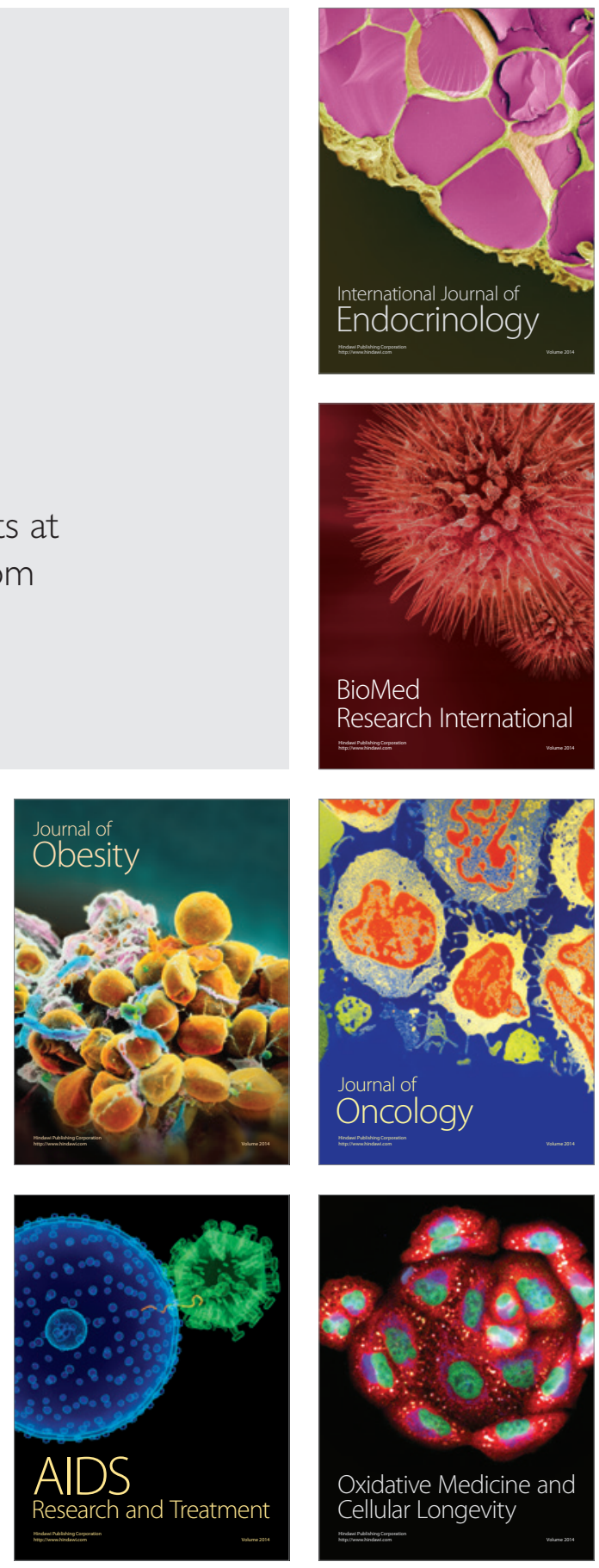